University of Nebraska - Lincoln

DigitalCommons@University of Nebraska - Lincoln

2012

\title{
Magnetic and structural transitions in the iron-chalcogenide high- Tc superconductor: K0.8Fe1.76Se2.00
}

\author{
W. N. Rowan-Weetaluktuk \\ McGill University \\ D. H. Ryan \\ McGill University, dhryan@physics.mcgill.ca \\ J. M. Cadogan \\ University of Manitoba \\ R. $\mathrm{Hu}$ \\ lowa State University, huhui@iastate.edu \\ S. L. Bud'ko \\ lowa State University, budko@ameslab.gov \\ See next page for additional authors
}

Follow this and additional works at: https://digitalcommons.unl.edu/usdoepub

Part of the Bioresource and Agricultural Engineering Commons

Rowan-Weetaluktuk, W. N.; Ryan, D. H.; Cadogan, J. M.; Hu, R.; Bud'ko, S. L.; and Canfield, Paul C., "Magnetic and structural transitions in the iron-chalcogenide high-Tc superconductor: K0.8Fe1.76Se2.00" (2012). US Department of Energy Publications. 347.

https://digitalcommons.unl.edu/usdoepub/347

This Article is brought to you for free and open access by the U.S. Department of Energy at DigitalCommons@University of Nebraska - Lincoln. It has been accepted for inclusion in US Department of Energy Publications by an authorized administrator of DigitalCommons@University of Nebraska - Lincoln. 


\section{Authors}

W. N. Rowan-Weetaluktuk, D. H. Ryan, J. M. Cadogan, R. Hu, S. L. Bud'ko, and Paul C. Canfield 


\title{
Magnetic and structural transitions in the iron-chalcogenide high- $T_{c}$ superconductor: $\mathrm{K}_{\mathbf{0 . 8}} \mathrm{Fe}_{\mathbf{1 . 7 6}} \mathrm{Se}_{\mathbf{2 . 0 0}}$
}

\author{
W. N. Rowan-Weetaluktuk, ${ }^{1}$ D. H. Ryan, ${ }^{1, a)}$ J. M. Cadogan, ${ }^{2}$ R. Hu, ${ }^{3}$ S. L. Bud'ko, ${ }^{3}$ \\ and P. C. Canfield ${ }^{3}$ \\ ${ }^{1}$ Physics Department and Centre for the Physics of Materials, McGill University, 3600 University Street, \\ Montreal, Quebec, H3A 2T8, Canada \\ ${ }^{2}$ Department of Physics and Astronomy, University of Manitoba, Winnipeg, Manitoba, R3T 2N2, Canada \\ ${ }^{3}$ Ames Laboratory, U.S. DOE and Department of Physics and Astronomy, Iowa State University, Ames, \\ Iowa 50011, USA
}

(Presented 3 November 2011; received 21 September 2011; accepted 1 November 2011; published online 29 February 2012)

\begin{abstract}
${ }^{57} \mathrm{Fe}$ Mössbauer spectroscopy was used to study single-crystals of $\mathrm{K}_{0.8} \mathrm{Fe}_{1.76} \mathrm{Se}_{2.00}$ from $6 \mathrm{~K}$ to $673 \mathrm{~K}$. At $6 \mathrm{~K}$, the hyperfine field $\left(B_{\mathrm{hf}}\right)$ is canted away from the $c$-axis by $18 \pm 3^{\circ}$. The temperature dependence of $B_{\mathrm{hf}}$ follows a spin wave model with a spin excitation gap of $9 \pm 1 \mathrm{meV}$. A sudden increase in the linewidth and a corresponding drop in $(1 / 2) e Q V_{z z}$ at $T_{N}=532 \mathrm{~K}$ are indications of strong coupling between the magnetic and structural transitions. (C) 2012 American Institute of Physics. [doi:10.1063/1.3673848]
\end{abstract}

\section{INTRODUCTION}

The iron chalcogenide $\mathrm{K}_{0.8} \mathrm{Fe}_{1.8} \mathrm{Se}_{2}$ is superconducting up to $T_{\mathrm{sc}}=30 \mathrm{~K}$ (Ref. 1) and antiferromagnetic up to $T_{N} \sim 559 \mathrm{~K}^{2,3}$ Neutron diffraction suggested that the $3.3 \mu_{B}$ iron moments are oriented parallel to the $c$-axis, ${ }^{2}$ while single-crystal Mössbauer data demonstrated a slight canting of the moments away from the $c$-axis. ${ }^{3}$ Single-crystal $\mathrm{x}$-ray diffraction showed that superconducting $\mathrm{K}_{y} \mathrm{Fe}_{2-x} \mathrm{Se}_{2}$ adopts an $I 4 / m$ structure below $T_{N}$, with a $(\sqrt{5} \times \sqrt{5} \times 1)$ iron vacancy ordering and that iron atoms fully occupy the $16 \mathrm{i}$ site. ${ }^{4}$ The collapse of the magnetism is associated with the loss of this vacancy ordering at a first order $I 4 / \mathrm{m} \rightarrow I 4 / \mathrm{mmm}$ magnetostructural transition. ${ }^{2,3}$

Single-crystal Mössbauer studies of $\mathrm{A}_{y} \mathrm{Fe}_{2}-{ }_{x} \mathrm{Se}_{2}(A=\mathrm{K}$, $\mathrm{Rb})^{3,5,6}$ all show a clear asymmetry in the intensity of the absorption peaks and a failure to account for this may lie behind the disagreement on the ordering direction with angles of $0^{\circ},{ }^{2} 18^{\circ},{ }^{3}$ and $40-45^{\circ}$ (Ref. 7) between the moments and the $c$-axis all being proposed. Li et al. ${ }^{5}$ have also claimed that the temperature dependence of the hyperfine field $\left[B_{\mathrm{hf}}(T)\right]$ exhibits evidence for a spin excitation gap (SEG) and that there is a drop in absorption associated with the superconducting transition. All of these issues are addressed here by analyzing the single-crystal Mössbauer spectra using a full Hamiltonian solution to the spectral shape.

\section{EXPERIMENTAL METHODS}

Single-crystals of $\mathrm{K}_{0.80} \mathrm{Fe}_{1.76} \mathrm{Se}_{2.00}$ were grown and characterized as previously described. ${ }^{8}$ The crystals were cleaved and mounted into spectrometers with the $\gamma$ beam aligned along the $c$-axis of the crystal mosaic. ${ }^{3}$ The Mössbauer spectra were collected using $50 \mathrm{mCi}{ }^{57} \mathbf{C o R h}$ sources and electromechanical drives using both constant acceleration

${ }^{a)}$ Electronic mail: dhryan@physics.mcgill.ca. and sine modes in conventional spectrometers. Calibration was done using an $\alpha$-Fe foil as a reference. Two closed-cycle He refrigerators were used in addition to a furnace for the high temperature data. One sample was cycled through the Néel temperature twice and another was cycled three times, spending over a week above $550 \mathrm{~K}$. Contrary to reports by Nowik et al., ${ }^{7}$ we observed no degradation of the samples below $673 \mathrm{~K}$ and the $I 4 / \mathrm{m} \rightarrow I 4 / \mathrm{mmm}$ structural transition appeared to be reversible.

The Mössbauer spectra were fitted using a nonlinear least squares minimization routine for ${ }^{57} \mathrm{Fe}$ single-crystal spectra employing the general static Mössbauer solution ${ }^{9}$ using a full Hamiltonian code.

\section{RESULTS}

The Mössbauer spectrum at $6 \mathrm{~K}$ (Fig. 1) was fitted using two components. The magnetic sextet, accounts for $86 \pm 1 \%$ of the spectrum and a paramagnetic impurity, the doublet, represents $14 \pm 1 \%$ of the spectrum. The deviation of the magnetic peak intensities from the $3: 2: 1: 1: 2: 3$ ratio and the paramagnetic peaks from the $1: 1$ ratio, is due to the use of oriented single-crystals as opposed to powder samples. The magnetic component has a clear asymmetry between the lines that correspond to the same $\Delta m_{I}$. This can best be seen in lines 2 and 5 , where line 2 is more intense than line 5 . This is an indication of a nonzero asymmetry parameter $(\eta)$. At $6 \mathrm{~K} \quad B_{\mathrm{hf}}=28.3 \pm 0.1 \mathrm{~T}, \quad(1 / 2) e Q V_{z z}=1.3 \pm 0.1 \mathrm{~mm} / \mathrm{s}$, $\eta=0.11 \pm 0.09$ and the angle, $\theta$, between $V_{z z}$ and $B_{\mathrm{hf}},=45 \pm 1^{\circ}$. For the paramagnetic component, we find that $V_{z z}$ is parallel to the $\gamma$-beam. These results are all consistent with values obtained for $\mathrm{Rb}_{0.8} \mathrm{Fe}_{1.6} \mathrm{Se}_{2.0}{ }^{6}$

The near vanishing of lines 2 and 5 indicates that the $\gamma$-beam and $B_{\mathrm{hf}}$ are nearly, but not quite, fully aligned, as has been previously reported. ${ }^{3,5,6}$ This contradicts the claim by Nowik et al. ${ }^{7}$ that the iron moments in $\mathrm{A}_{0.8} \mathrm{Fe}_{2-y} \mathrm{Se}_{2}(A=\mathrm{K}$, 


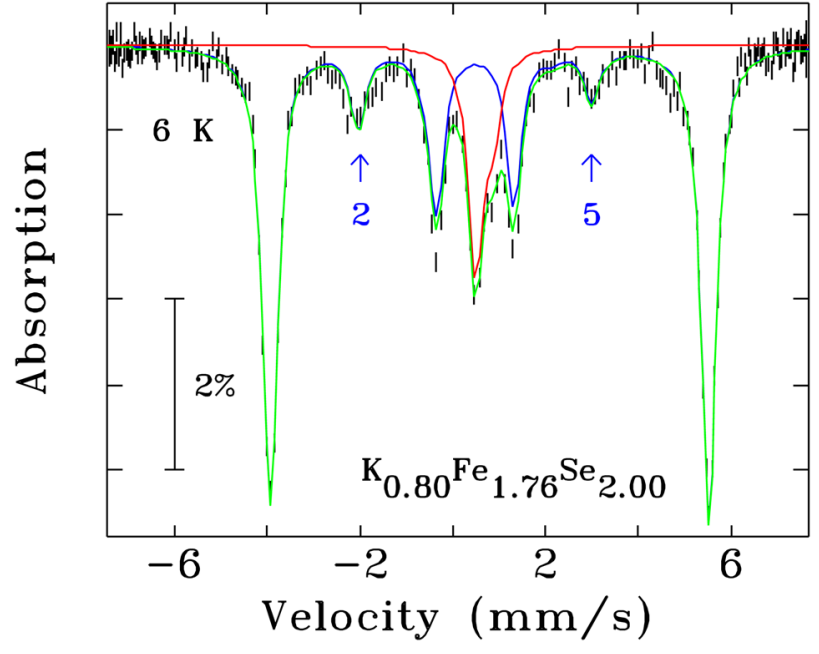

FIG. 1. (Color online) Mössbauer spectrum at $6 \mathrm{~K}$ of $\mathrm{K}_{0.80} \mathrm{Fe}_{1.76} \mathrm{Se}_{2.00}$. The spectrum was fitted using a full Hamiltonian code. The blue sextet is the magnetic component, the red doublet is an impurity and the solid green line through the data is the sum of the two components. Lines 2 and 5 of the magnetic sextet are marked.

$\mathrm{Rb}$ and $\mathrm{K} / \mathrm{Tl}$ ) are tilted by $40-45^{\circ}$ away from the $c$-axis. A conclusion arrived at by using powders and assuming that $V_{z z}$ points along the c-axis. However, the low point symmetry of the 16i site (1) allows $V_{z z}$ to make an arbitrary angle with the $c$-axis. Using single-crystals, unlike powders, permits the direct measurement of the $18 \pm 3^{\circ}$ angle between the $\gamma$-beam and $B_{\mathrm{hf}}$. This angle comes from three independent mosaics run on different spectrometers and exceeds any possible misalignment. While we cannot distinguish between uniaxial canting and some form of conical spin arrangement, there is definitely a small canting of $B_{\mathrm{hf}}$, and hence the iron moments, away from the $c$-axis. This same canting has now been seen in both the potassium ${ }^{3}$ and rubidium ${ }^{6}$ compounds and suggests that the neutron diffraction results ${ }^{2,10}$ need to be re-evaluated.

The low temperature dependence of the hyperfine field is shown in Fig. 2. A $T^{3 / 2}$ spin wave model shown by the dotted line in Fig. 2 fails to reproduce adequately $B_{\mathrm{hf}}(T)$ so we included a spin excitation gap:

$$
B_{\mathrm{hf}}(T)=B_{\mathrm{hf}}(0)\left(1-C T^{3 / 2} \sum_{k=1}^{\infty} \frac{e^{-\Delta E / k_{B} T}}{k^{3 / 2}}\right),
$$

where $B_{\mathrm{hf}}(0)$ is the hyperfine field at $0 \mathrm{~K}, \mathrm{C}$ is the spin stiffness constant, $\Delta E$ is the energy of the SEG and $k_{B}$ is Boltzmann's constant. ${ }^{11}$ A previous study claimed a gap of $\sim 5 \mathrm{meV}^{5}{ }^{5}$ here the gap was found to be $9 \pm 1 \mathrm{meV}$ with a spin stiffness constant of $18 \pm 1 \times 10^{-6} \mathrm{~K}^{-3 / 2}$. The summation was limited to the first 19 terms of the series. The difference between the infinite series and our limited summation is less than $0.001 \%$.

The integrated spectral area, $\zeta$, is shown in Fig. 3. $\zeta$ s for different samples in different spectrometers have been combined by scaling data taken at a common temperature in order to cover the full temperature range of the experiments. Li et al. ${ }^{5}$ claimed a $\sim 10 \%$ drop in $\zeta$ associated with the superconducting transition and a similar one below the

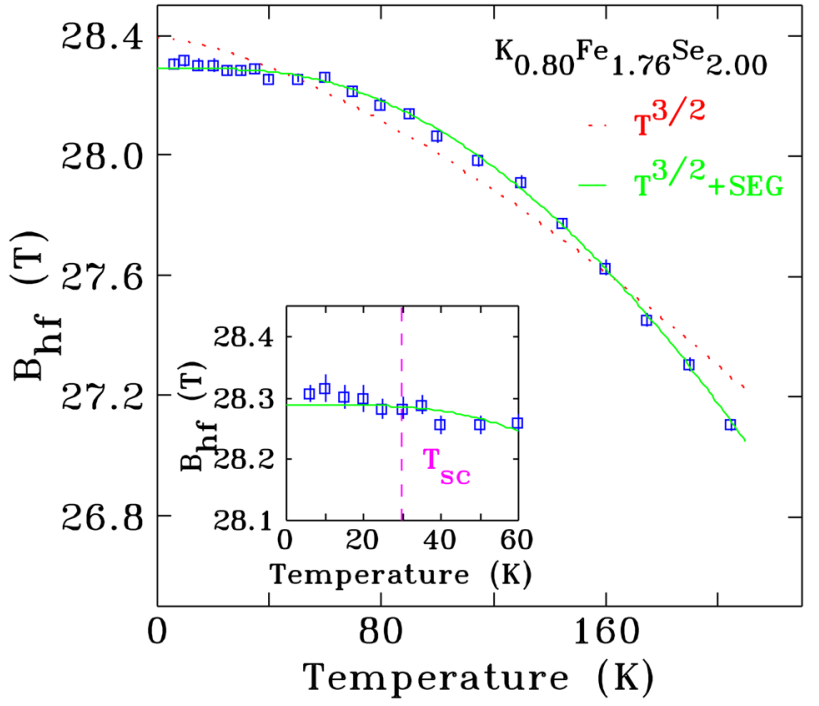

FIG. 2. (Color online) The hyperfine field $\left(B_{\mathrm{hf}}\right)$ of the antiferromagnetic subspectrum of $\mathrm{K}_{0.80} \mathrm{Fe}_{1.76} \mathrm{Se}_{2.00} . B_{\mathrm{hf}}(T)$ was fitted using a $T^{3 / 2}$ law (dotted red line), and a $T^{3 / 2}$ law with a SEG (solid green line) up to $200 \mathrm{~K}$. The SEG was found to be $9 \pm 1 \mathrm{meV}$. The inset shows $B_{\mathrm{hf}}(T)$ close to the superconducting transition $\left(T_{\mathrm{sc}}\right)$, marked as the dashed magenta line.

transition. No such drops were observed here, as can be seen in the inset of Fig. 3. Since there is no structural transition or other behavior causing lattice softening associated with the superconducting transition, no drop in $\zeta$ is expected. Fitting of $\zeta(\mathrm{T})$ to a Debye-Waller model between 6 and $673 \mathrm{~K}$ (Fig. 3), yields $\theta_{D}$ of $195 \pm 2 \mathrm{~K}$, typical for an intermetallic.

The development of a soft phonon mode near the $I 4 / \mathrm{m} \rightarrow I 4 / \mathrm{mmm}$ structural transition could lead to a reduction in the $f$-factor. ${ }^{12}$ However, no drop in absorption was observed. The small break near $T_{N}$ in Fig. 3 is consistent with a fitting artifact (there are rapid changes in both the spectral form and linewidth near $T_{N}$ ) and would be followed by a recovery if it were indeed due to a soft mode.

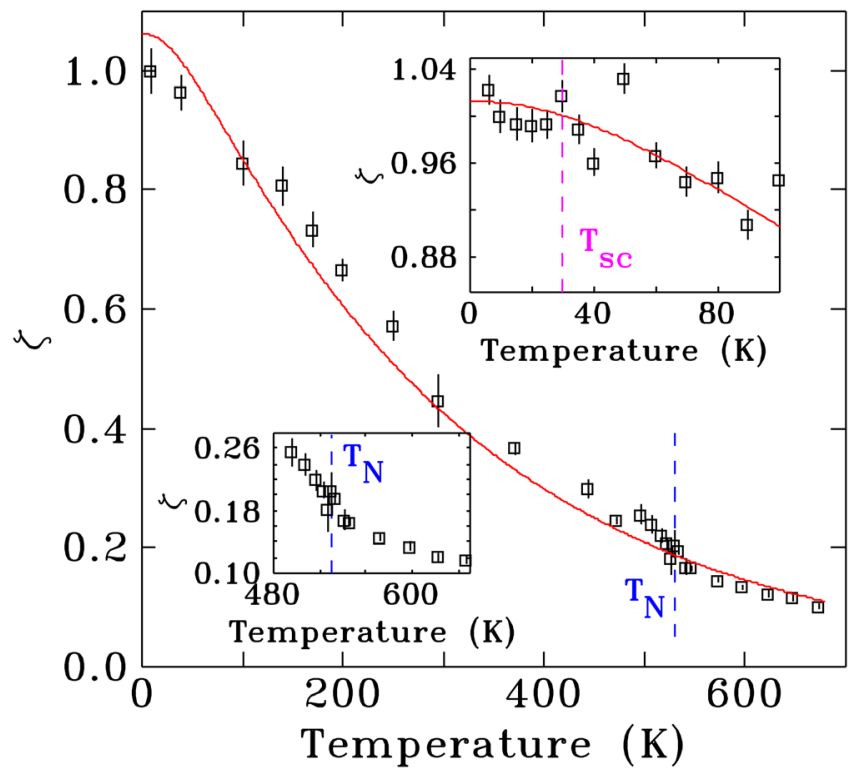

FIG. 3. (Color online) The integrated spectral area, $\zeta$, fitted using a DebyeWaller model giving $\theta_{D}=195 \pm 2 \mathrm{~K}$. The insets show $\zeta$ : (top right) near the superconducting transition, the magenta line, and (bottom left) the magnetic transition, the blue line. 


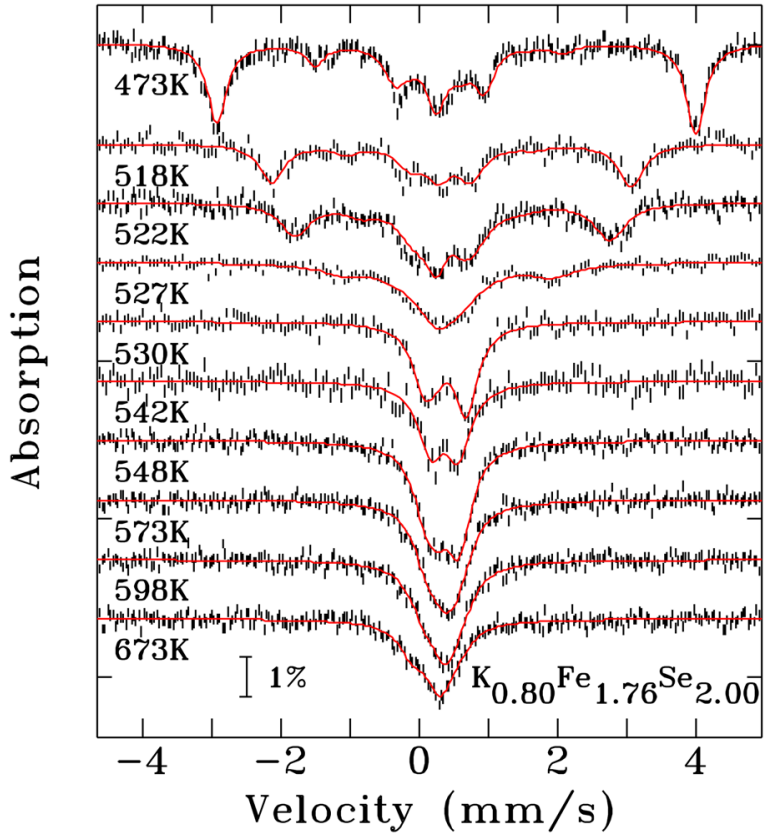

FIG. 4. (Color online) Mössbauer spectra of $\mathrm{K}_{0.80} \mathrm{Fe}_{1.76} \mathrm{Se}_{2.00}$. The fits to the spectra, made using a full Hamiltonian solution, are shown as solid red lines.

The high temperature Mössbauer spectra are shown in Fig. 4. The magnetic transition is obvious from the collapse of the outer (magnetic) peaks, accompanied by the growth of a paramagnetic doublet. As we heat through $T_{N}$ there is a marked reduction in $(1 / 2) e Q V_{z z}$ (Fig. 5), the new paramagnetic component starts to appear at least $10 \mathrm{~K}$ below $T_{N}$ and the transformation in $(1 / 2) e Q V_{z z}$ is not complete until $10 \mathrm{~K}$ above $T_{N}$ (upper inset to Fig. 5). The linewidth, $\Gamma$, increases dramatically and then recovers (lower inset to Fig. 5). These changes indicate that the structural transition from $I 4 / \mathrm{m}$ to $I 4 / \mathrm{mmm}$ is closely linked to the magnetic transition. It starts before the magnetism collap-

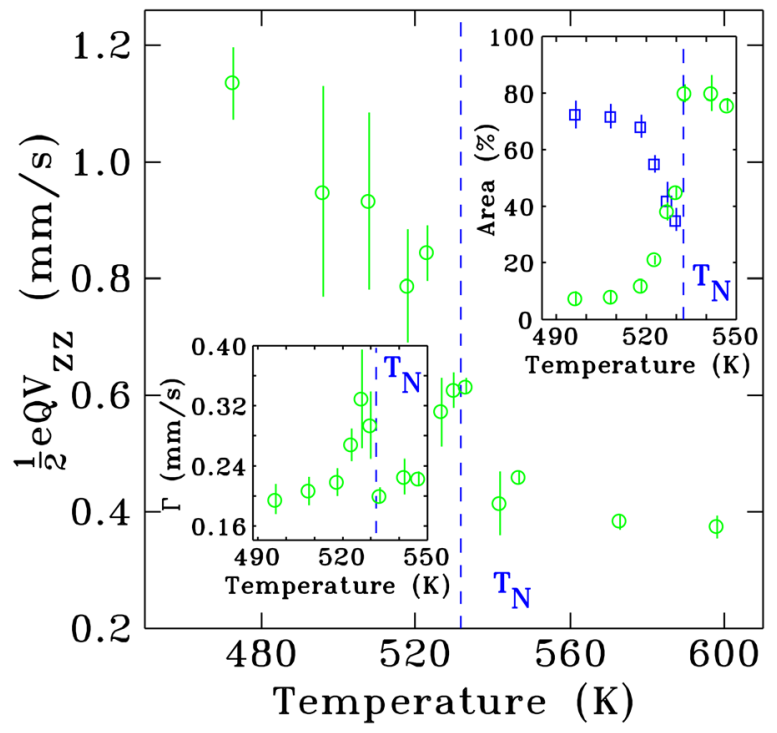

FIG. 5. (Color online) (1/2)eQV $V_{z z}$ of $\mathrm{K}_{0.80} \mathrm{Fe}_{1.76} \mathrm{Se}_{2.00}$ determined from the Mössbauer spectra shown in Fig. 4. The lower inset shows the spectral linewidth $(\Gamma)$ while the upper inset shows the fractional areas of the magnetic (blue $\square$ ) and paramagnetic (green $\bigcirc$ ) components. The Néel temperature, $T_{N}=532 \pm 2 \mathrm{~K}$, is marked by the dashed blue line. ses and likely causes the collapse. The increase in linewidth suggests a rapid increase in disorder at the iron site, while the reduction in $(1 / 2) e Q V_{z z}$ is consistent with an increase in symmetry associated with the structural transition. Although neutron diffraction data have been interpreted as showing a gap of $\sim 20 \mathrm{~K}$ between the $\mathrm{T}_{N}$ and $T_{S},{ }^{2,10}$ the results in Fig. 5 demonstrate that the first stages of the $I 4 / \mathrm{m} \rightarrow I 4 / \mathrm{mmm}$ transition start well below $T_{N}$ and continue after the magnetic order is lost. The two transitions are essentially coincident and the changes in crystal structure drive the loss of magnetic order.

\section{CONCLUSIONS}

The iron moments in $\mathrm{K}_{0.80} \mathrm{Fe}_{1.76} \mathrm{Se}_{2.00}$ are canted away from the $c$-axis by $18 \pm 3^{\circ}$ below $T_{N}$. Below $\sim 200 \mathrm{~K}, B_{\mathrm{hf}}$ follows a gapped spin wave model with a SEG of 9(1) $\mathrm{meV}$. Material degradation is not an intrinsic property of the $I 4 / \mathrm{m} \rightarrow I 4 / \mathrm{mmm}$ transition, rather it stems from reactions with mounting materials or contaminated vacuum. The $I 4 / \mathrm{m} \rightarrow I 4 / \mathrm{mmm}$ structural transition is coincident with the loss of antiferromagnetic order and likely plays a causal role in the loss of magnetic ordering.

\section{ACKNOWLEDGMENTS}

This work was supported by grants from the Natural Sciences and Engineering Research Council of Canada, and Fonds Québécois de la Recherche sur la Nature et les Technologies. JMC is supported by the Canada Research Chairs program. R.H. and P.C.C. are supported by AFOSR-MURI Grant No. FA9550-09-1-0603. S.L.B. and P.C.C. are also supported by the U.S. DOE BES DMSE. Ames Laboratory is operated for the U.S. DOE by ISU under Contract No. DEAC02-07CH11358. We are grateful to G. Wortmann (MaxPlanck Institute for Chemistry) for suggesting to us that the asymmetry in the magnetic spectra was the result of a nonaxially symmetric quadrupole interaction $(\eta \neq 0)$.

${ }^{1}$ J. Guo, S. Jin, G. Wang, S. Wang, K. Zhu, T. Zhou, M. He, and X. Chen, Phys. Rev. B 82, 180520 (2010).

${ }^{2}$ B. Wei, H. Qing-Zhen, C. Gen-Fu, M. A. Green, W. Du-Ming, H. JunBao, and Q. Yi-Ming, Chin. Phys. Lett. 28, 086104 (2011).

${ }^{3}$ D. H. Ryan, W. N. Rowan-Weetaluktuk, J. M. Cadogan, R. Hu, W. E. Straszheim, S. L. Bud'ko, and P. C. Canfield, Phys. Rev. B 83, 104526 (2011).

${ }^{4}$ P. Zavalij et al., Phys. Rev. B 83, 132509 (2011).

${ }^{5}$ Z. Li, X. Ma, H. Pang, and F. Li, arXiv:1103.0098v1 (2011).

${ }^{6}$ V. Ksenofontov, G. Wortmann, S. Medvedev, V. Tsurkan, J. Deisenhofer, A. Loidl, and C. Felser, Phys. Rev. B 84, 180508 (2011).

${ }^{7}$ I. Nowik, I. Felner, M. Zhang, A. F. Wang, and X. H. Chen, Supercond. Sci. Technol. 24, 095015 (2011).

${ }^{8}$ R. Hu, K. Cho, H. Kim, H. Hodovanets, W. E. Straszheim, M. A. Tanatar, R. Prozorov, S. L. Bud'ko, and P. C. Canfield, Supercond. Sci. Technol. 24, 065006 (2011).

${ }^{9}$ C. J. Voyer and D. H. Ryan, Hyperfine Interac. 170, 91 (2006).

${ }^{10}$ F. Ye, S. Chi, W. Bao, X. F. Wang, J. J. Ying, X. H. Chen, H. D. Wange, C. H. Dong, and M. Fang, Phys. Rev. Lett. 107, 137003 (2011).

${ }^{11}$ V. Senz, R. Röhlsberger, J. Bansmann, O. Leupold, and K.-H. MeiwesBroer, New J. Phys. 5, 47 (2003).

${ }^{12}$ W. N. Rowan-Weetaluktuk, D. H. Ryan, A. O. Sushkov, S. Eckel, S. K. Lamoreaux, O. P. Sushkov, J. M. Cadogan, M. Yethiraj, and A. J. Studer, Hyperfine Interac. 198, 1 (2010). 